\title{
Usability evaluation of newspaper-on- dvd (np-dvd) of the Rizal library, Ateneo de Manila University
}

\author{
Engracia S. Santos \\ Ateneo de Manila University \\ Rizal Library Special Collections Building \\ Katipunan Avenue, Loyola Heights \\ 1108 Quezon City \\ Philippines \\ Cell: +632 9175876936 \\ esantos@ateneo.edu
}

\begin{abstract}
:
The primary objective of this study is to determine the usability of the Newspaper-onDVD project of the Rizal Library, Ateneo de Manila University and to make recommendations that will help improve the system and expand its usage. In 2000, Library started transforming the preserved newspaper from microfilm to digital images. The library then provided an easier mode of access by providing a search tool that will link the index to the images and to the printers. A descriptive evaluative research method through usability testing was used in this study. Ten representative students were asked to complete a series of tasks using NP-DVD. Based on the test, the researcher was able to identify usability problems and recommended futures actions to enhance the system. These problems characterize the difficulties users face while using library search tools available not only in the libraries but also in the internet.
\end{abstract}

Keywords: Usability, User testing, Library search tools

\section{Introduction}

For almost four hundred years, people have been reading newspapers all over the world. A newspaper is a resource for history, fashion and art, commerce and values, culture, politics and a thousand significant things that people want to know (Ronan, 2006). Political chaos caused alarm in the peace and order situation of the Philippines in the late 1960s. This incident, plus the growing need to look for newspapers, gave birth to the Current Newspaper in Microfilm project. This project was supported by the Philippine Library Material Project Foundation (Lacanieta, 2000). However, problem with storage, subscription cost, preservation, and access continues in schools and universities in the different islands of the Philippines. In 2000, digital imaging promised a cheaper alternative to microfilming by providing graphical images that can be viewed in desktop computers, which is available even in small libraries (United States Newspaper Project, 2005).

With this technological developments, Rizal Library developed an application system that may be used by library to access the archive of daily newspaper. A memorandum of agreement with four major dailies, Philippine Daily Inquirer, The Philippine Star, The Manila Times, and Malaya, allowed the Library to archive their newspapers and distribute the digital copy to libraries needing it at minimal cost. The system will allow the linking of the Computerized Index to Philippine Periodical Articles (CIPPA) to the images. The index includes the title of the article, its author, subject, publisher, date, and page number (Mariano, 2005). The system allows basic and advance search facility using a combination of 
exhaustive title index and selective subject index from CIPPA database; retrieval of the actual image of the page; and a facility to print the page or a portion of the page (see Figure 1).

This purpose of this study is to evaluate the performance of NP-DVD in terms of task time, completion rate, error rate, memorability rate, and satisfaction rate. In addition, the study identified ways to further enhance improve the system.

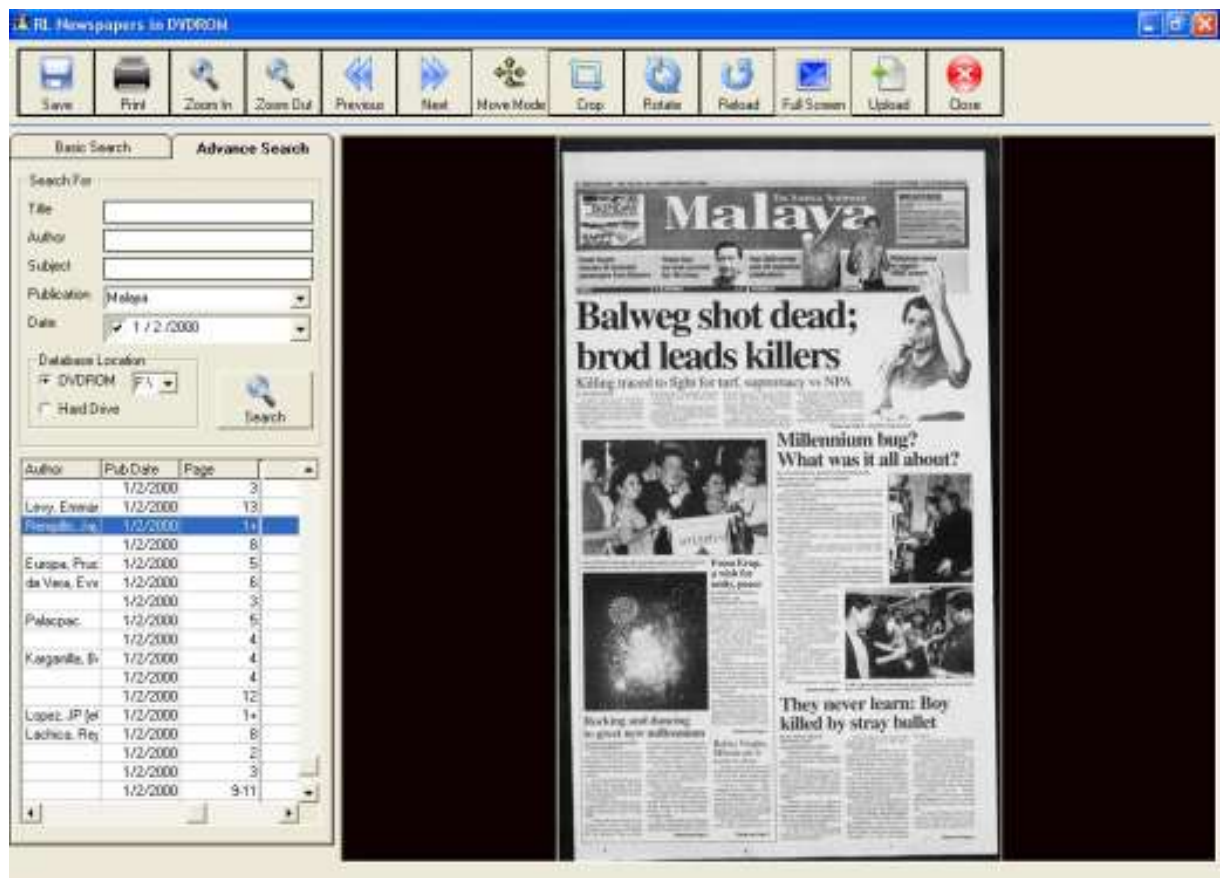

Figure 1: Screen shot from the NP-DVD

\section{Methodology}

The study was based on the combination of the following theories. Nielsen's (2012) defined usability as the measure of quality of the user experience when interacting with something software application, or any other device that the user can operate. Lewis (2008) uses factor analysis to come up with a general construct of usability with two underlying factors, namely the objective factor and the subjective factor. The objective factor measures effectiveness and efficiency by task times, completion rate, and errors. While the subjective factor is measured by the post-test satisfaction score. Lastly, Nielsen (2000) and Landauer (1995) explained that the successful usability testing focused on giving specific tasks to five users and measures the following attributes of usability: Learnability, Efficiency, Memorability, Error, and Satisfaction.

For this study, a series of five task scenarios were prepared for the respondents:

- Task Scenario 1: To test if NP-DVD is easy to use or not

- Task Scenario 2: To test if the user can perform a basic search task

- Task Scenario 3: To test if the user can perform an advance search task

- Task Scenario 4 and Scenario 5: To test if the user can make full use of the system on his/her own (these tasks were flexible so users can use several strategies to achieve their goal) 
Pre-test activities include document analysis; personal interviews with the systems developer; personal interviews with the librarians; and pilot testing. A standard orientation guide was also prepared to ensure that the same basic information was given to the test participants. The pre-test questionnaire was used to gather the baseline data about the participants while post task questionnaires were administered after completion of each task.

\section{Participants}

For the purpose and design of this study, the participants must be selected from NP-DVD subscribers who have a complete set of DVDs to be able to provide standard setting for the usability test. Of the 54 NP-DVD subscribers, only seven are subscribed to the full system. Of which, only three are located in Metro Manila. However, one of the organizations requested to be excluded in the study because they are not yet fully implementing the use of NP-DVD. The two universities agreed to set up an area in the library with almost the same physical set-up to provide standard settings for the usability test. Lastly, since most minor subjects, such as history, sociology and humanities, are offered in first and second year levels, the ten representative users are selected from $1^{\text {st }}$ year or $2^{\text {nd }}$ year students who are first time users of the NP-DVD.

\section{Result of the Usability Test Task Scenario 1: To test if NP-DVD is easy to use or not}

Fifty percent of the respondents rated the system as "moderately easy," 30 percent of the respondents rated it "moderately difficult and 20 percent rated it "easy" (see Figure 2). It was apparent in the result, that even though the participants are first time users, 80 percent of them were able to complete the task without difficulty. While those who rated the system as "moderately difficult" encountered errors (e.g., loading DVD) while performing the task.

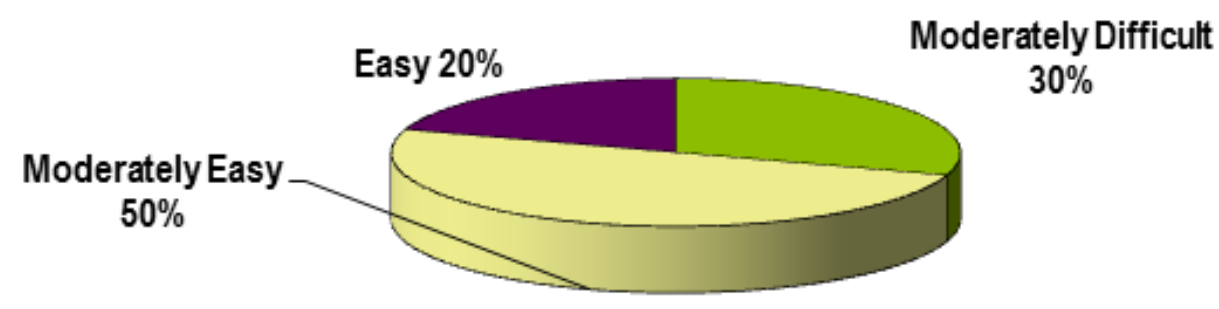

Figure 2: Rating on using the NP-DVD after completing Task 1

Majority of them (70\%) rated it "moderately easy." On the other hand, 20 percent rated it "neither easy nor difficult" while 10 percent rated it "easy" (see Figure 3). As an exploratory task for first time users, layout of the screen and navigation tools plays a vital role in the usability of the system. The result illustrates that 80 percent participants finds the icons on the screen easily. These icons are important in performing the activities required in the task. 


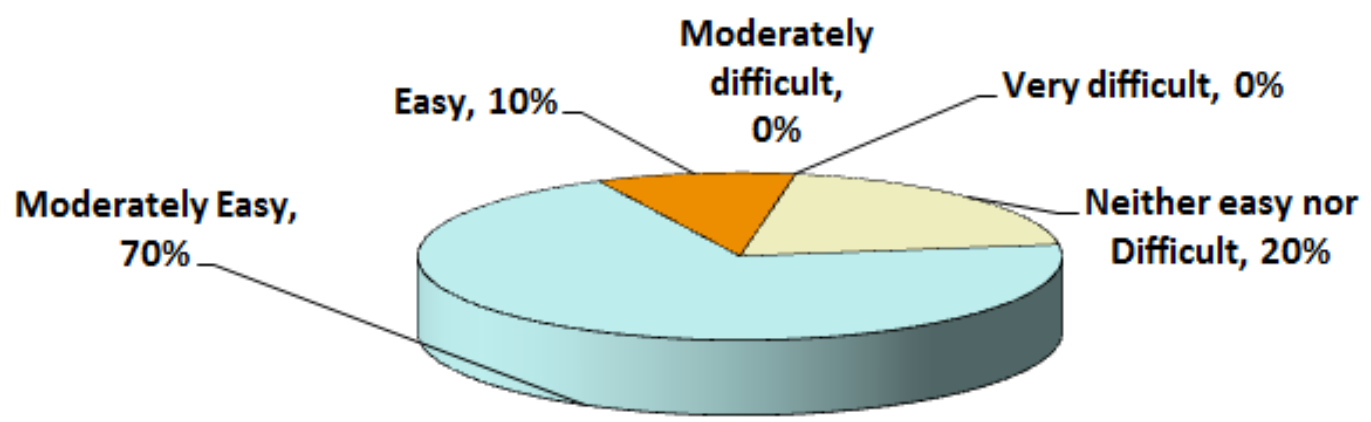

Figure 3: Rating on finding the icons on the screen

Eighty percent found the wording "very clear" while 20 percent rated it "moderately clear." Thus, it can be assumed that most of the respondents understand the wordings because the said program used words and phrases which are easy to understand and are very clear. Familiarity with the wordings used in the system facilitates usability of the system (see Figure 4).

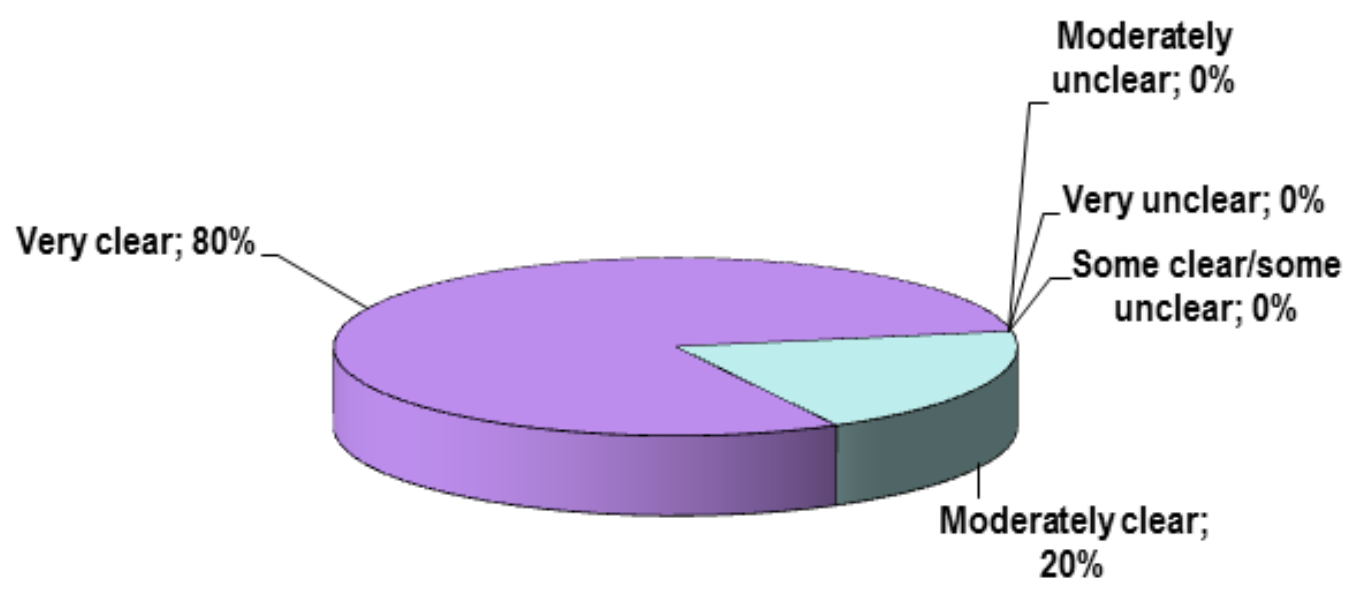

Figure 4: Rating on the wordings used (if it is easy to understand)

When it comes to the part of the screen that catches the attention of the respondents, the survey shows majority of them $(60 \%)$ were attracted by the "icons found on the screen." On the other hand, 20 percent of them indicated that their attention was caught by the display area or the middle part of the screen. Others' attention were caught by the "search panel/side" or search tabs (20\%; see Figure 5). 


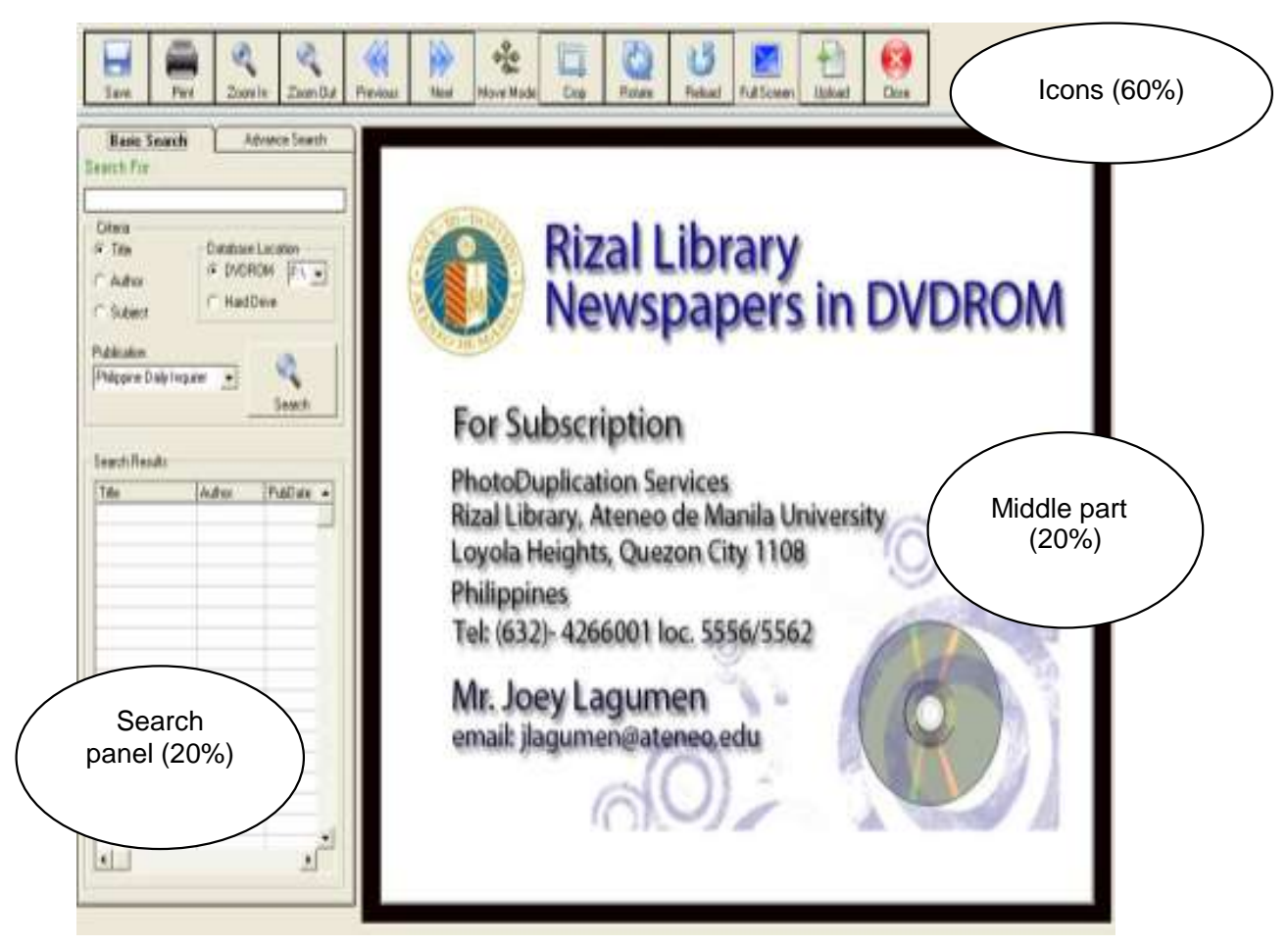

Figure 5: Part of the screen which catches the attention of the respondents

The respondents cited the following reasons why they noticed the "icons": it has pictures; its big enough; and its location on screen. Then, the respondents quoted the following as reasons why the saw the "middle part": it has a picture/some information about the product and letters have designs and various colors. Lastly, the respondents like the "search panel" because it looks like an ordinary search page that we see in other application programs from the internet.

In Task 1, User 1 was the fastest with an average time of 0:04:40 minutes in performing the eight activities (Open, Basic Search, Search, Open, DVD, Enlarge, Write, and Close) while the slowest among the respondents was User 6 with an average time of 0:06:25 minutes. Almost all of them completed (90\%) the eight activities even though they are first time users of the system. This reflects that the navigation, layout and terminology used enables the respondents to complete their task.

Four among the ten users got an error rate of 0.38 in performing the activities while two respondents got the highest error rate with an error rate of 1.25 . This result is attributed to the fact that most of the participants found the icons needed to perform the task moderately easy/easy. Also, all of them found the wordings moderately clear and very clear.

The average memorability score that the respondents got range from 2.88 to 3.63 or "proficient" to "very proficient." The high rating given by the observer showed that majority of the respondents was able to remember and perform the activities in Task 1 with ease. The reason for these is the use of icons and other user interfaces that is common in other applications packages.

Task Scenario 2: To test if the user can perform a basic search task Majority of them rated the system "moderately easy" in doing basic search while 10 percent rated it "neither easy nor difficult" (see Figure 6). The participants observed the similarity of 
the search panel to other applications. It can be presumed that the exposure of the participants to different internet and computer applications also helped in the performance of the activities.

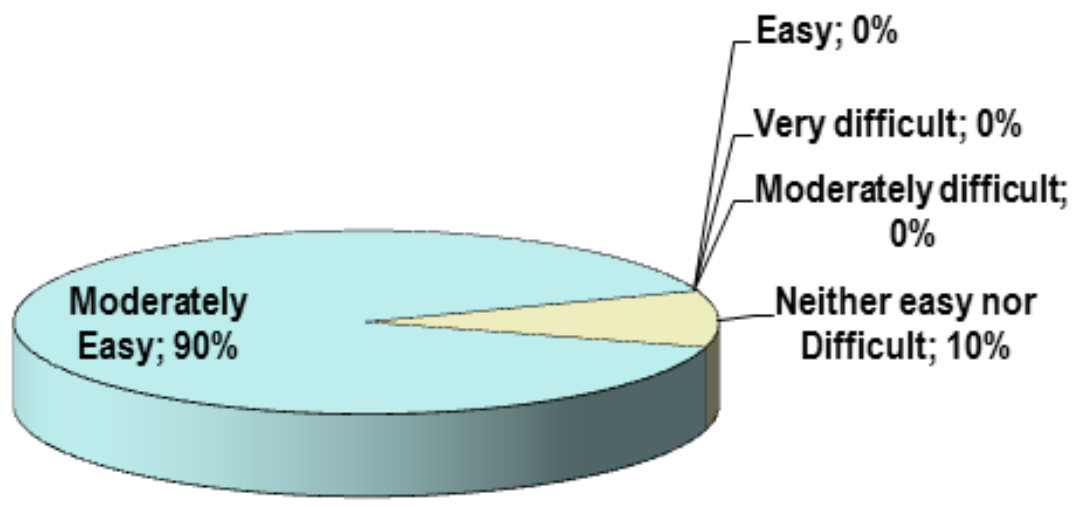

Figure 6: Difficulty level in performing basic search

Ninety percent mentioned "the searching process/typing key words" as the easiest. On the other hand, 10 percent cited "getting the DVD" (see Figure 7). This result also shows that the NP-DVD is achieving one of its objectives by providing a facility to easily search for the contents of the newspaper.

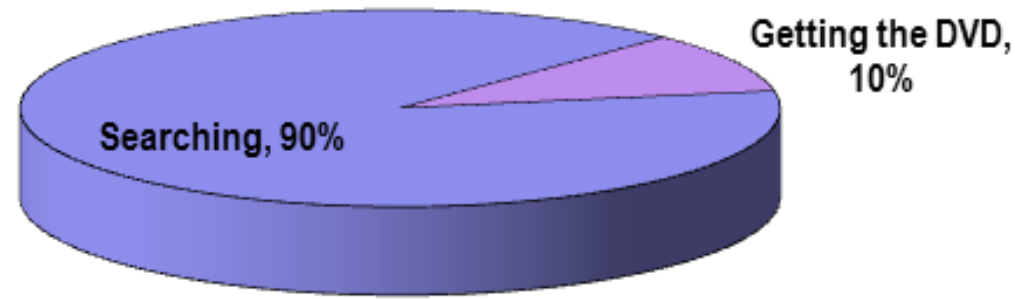

Figure 7. Easiest thing to do or understand after Task 2

Most of them found "the cropping process" as difficult. On the other hand, 20 percent cited "loading the DVD" and 10 percent cited "list down result." Those participants who have difficulty in the cropping process were confused with the use of move and select mode (see Figure 8). Also, they expected the crop symbol ( crop mode is active. Twenty percent of the respondents had difficulty in loading the DVD because they accidentally pressed other buttons while waiting for the DVD drive to read and load the database. Also, the automatic read function of the computers opens the DVD pop up screen, thus the users invoke another program of the computer. Lastly, the participants complained of writing down the result of the test on the answer sheet. 


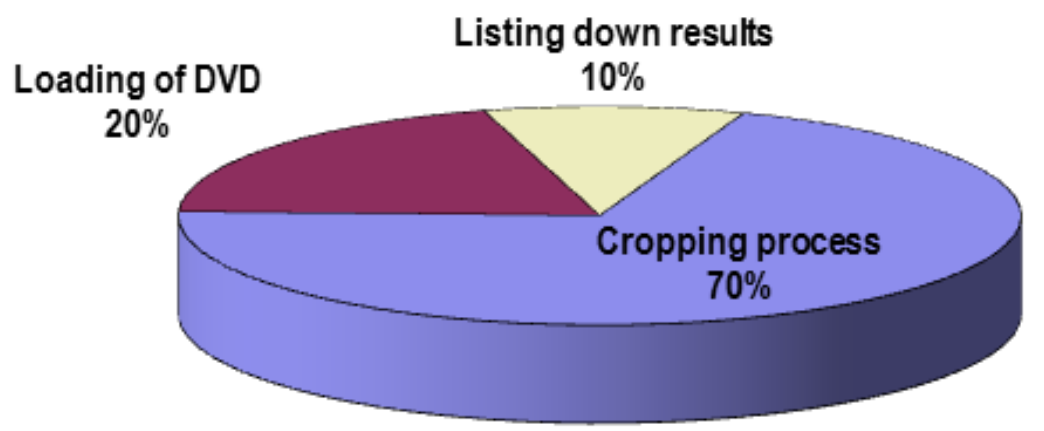

Figure 8. Difficult thing to do or understand

Due to the difficulty in the cropping process, 50 percent of the participants suggested changing it (see Figure 9). The suggestion is the use of a common cropping symbol (\$). The other half of the participants focused on the errors encountered while loading the DVD. The participants suggested loading the database of images in a hard disk or make it available in a network. The cropping error is an error within the system while the second error is due to the time elapsed while the computer reads the DVD and therefore considered external to the system.

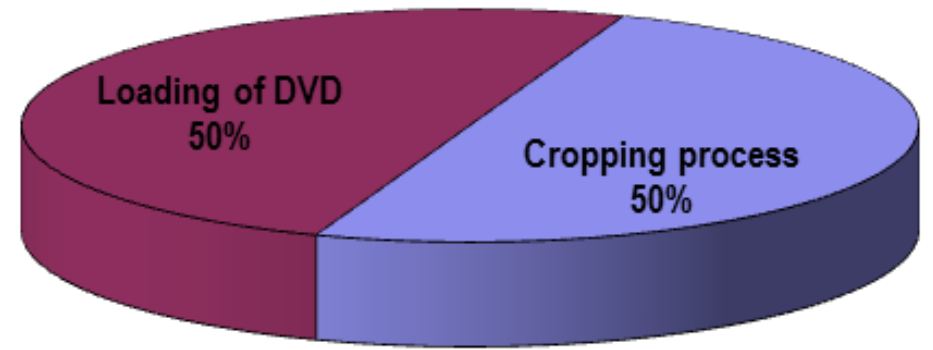

Figure 9. Changes to be made to make Task 2 easier

User 5 is the fastest with an average time of 0:05:10 minutes in performing the eight activities (Open, Basic Search, Search, Open, DVD, Enlarge, Write, and Close) while the slowest among the respondents was User 7 with an average time of 0:06:40 minutes. Other respondents' average time ranges from 0:05:30 to 0:06:30. The results of the activities revealed that almost half ( 2.57 mins. or $43 \%)$ of the average time $(6.0$ mins.) consumed by the participants in performing two activities, namely, loading the DVD and cropping process. Though the result of the test seemed to be fast enough, if these two activities will be improve the task time of the participants will be greatly enhanced.

Majority of them got a completion rate of 1 . This is with the exception of User 1 , who was not able to finish because she panicked and instead of focusing on the task ahead of her she called the library assistant to help. One among the ten users got an error rate of 0.20 in performing the activities while one respondent got the highest error rate with an error rate of 1.10. This suggests that the representative users who have undergone the usability testing 
were familiar enough with the steps in performing basic search tasks. The memorability rate of Task 2 range from 3.70 to 4.60 . Said result was attributed to the familiarity of the representative users with the steps in performing basic search tasks.

\section{Task Scenario 3: To test if the user can perform advance search task}

Majority of them rated it "moderately easy" though 10 percent rated it "neither easy nor difficult" (see Figure 10). The result of the test showed that the participants did not feel the degree of difficulty from basic to advance search. We can speculate that this is because the two tasks have almost the same activities.

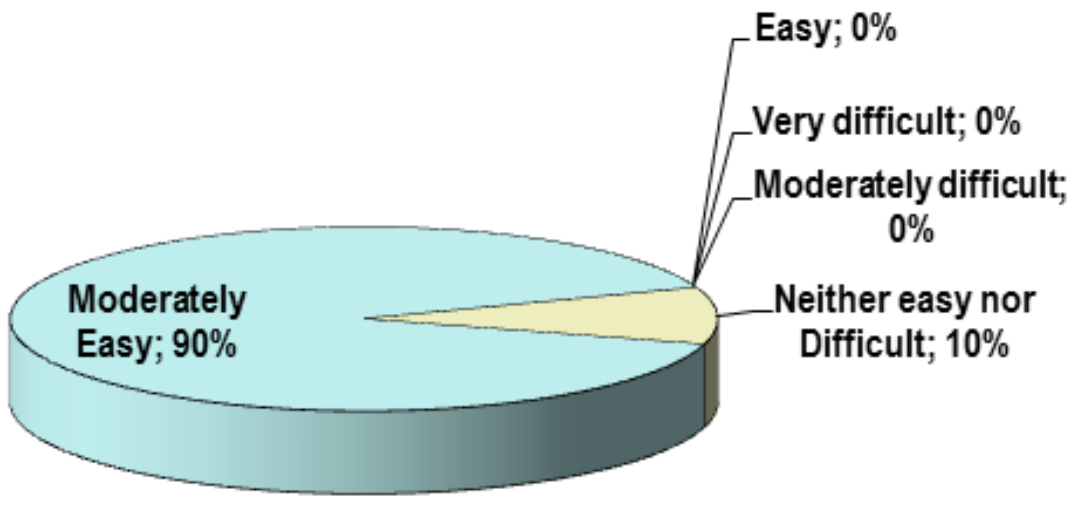

Figure 10: Difficulty level in performing advance search

Seventy percent mentioned "the searching process/typing key words" as the easiest thing to do. Others cited the following: cropping \& zooming $(20 \%)$ and printing $(10 \%$; see Figure 11$)$. The test result shows that after performing Task 3, some (20\%) respondents find cropping/zooming and printing easy to do. Thus, it shows how the participants easily adapted to the NP-DVD.

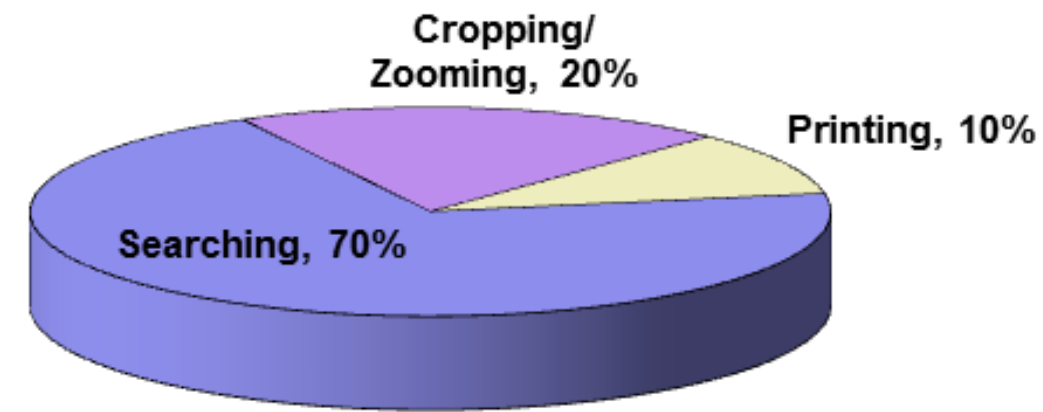

Figure 11: Easiest thing to do or understand after Task 3

Majority of them mentioned "the problem in loading the DVD" as difficult. The respondents encountered the same difficulty (i.e., they accidentally pressed other buttons while waiting for the DVD drive to read and load the database). Still, some of the participants (20\%) have the same difficulty in the cropping process as in the previous task (see Figure 12). 


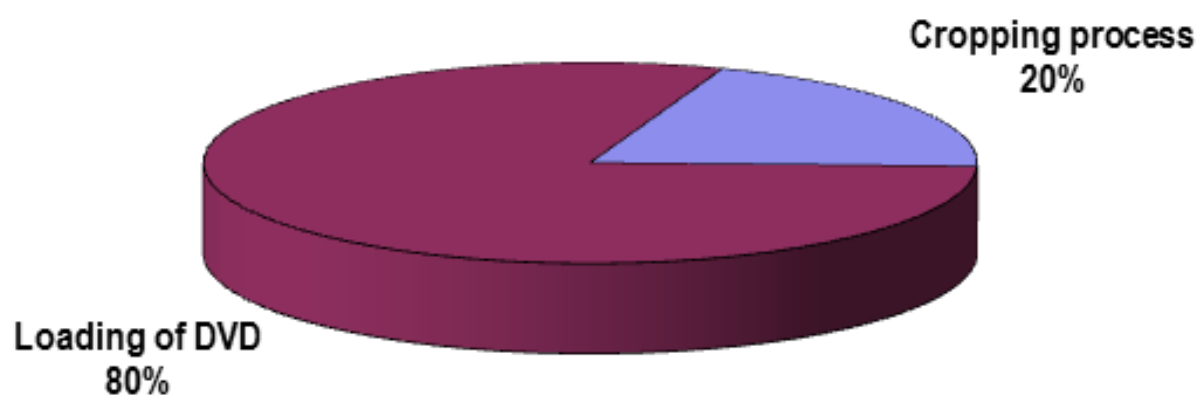

Figure 12. Difficult thing to do or understand in Task Scenario 3

When it comes to the question "What will you change to make the task easier?" five mentioned "none" while the rest cited the following: loading the DVD (30\%) and cropping $(20 \%)$. The percentage of these two things that the respondents wanted to change in the system dropped from 50 percent to 30 percent and 50 percent to 20 percent, respectively. It also reflects how fast the participants learned to use the system after the third task (see Figure 13).

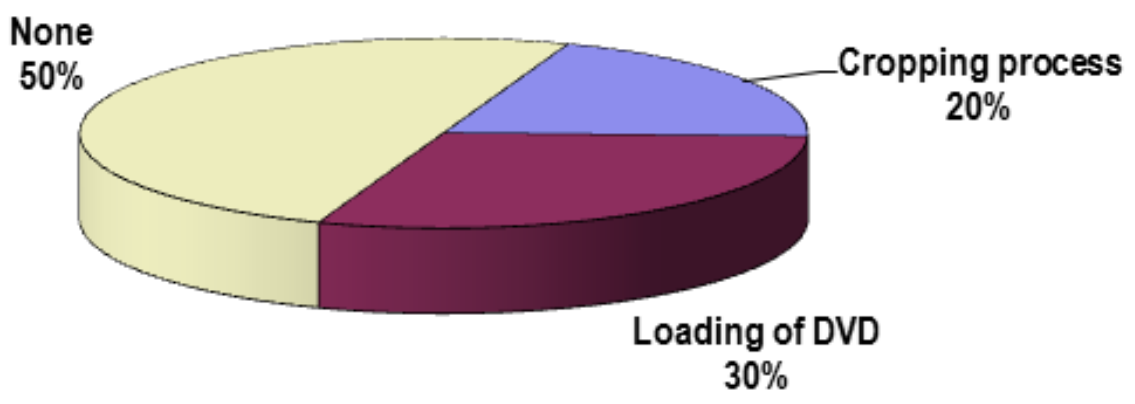

Figure 13: Changes to be made to make Task 3 easier

User 6 was the fastest with an average time of five minutes in performing the eight activities (Open, Basic Search, Search, Open, DVD, Enlarge, Write, and Close) while the slowest among the respondents were Users 4 and 8 with an average time of six minutes. The other respondents' average time ranges from 0:05:10 to 0:05:55 minutes. Results can be attributed to the variation of the search process from simple search to advance search. Two among the ten users got a zero error rate in performing the activities while two respondents got the highest error rate with an error rate of 0.40 . The test showed that the users can perform an advance search task with a higher degree of difficulty with a very minimal error rate. The memorability rate that the respondents got range from 4.20 to 5.00 .

\section{Task Scenario 4: To test if the user can make full use of the system using the most frequently used style}

Fifty percent rated it "neither easy nor difficult." On the other hand, 30 percent rated it "moderately easy while 20 percent rated it "easy" (see Figure 14). The result can mean that creativity of participants and knowledge of computer application programs are an advantage when they are not familiar with the task given them. Their skill is shown when they are navigating to the different pages of the newspaper. 


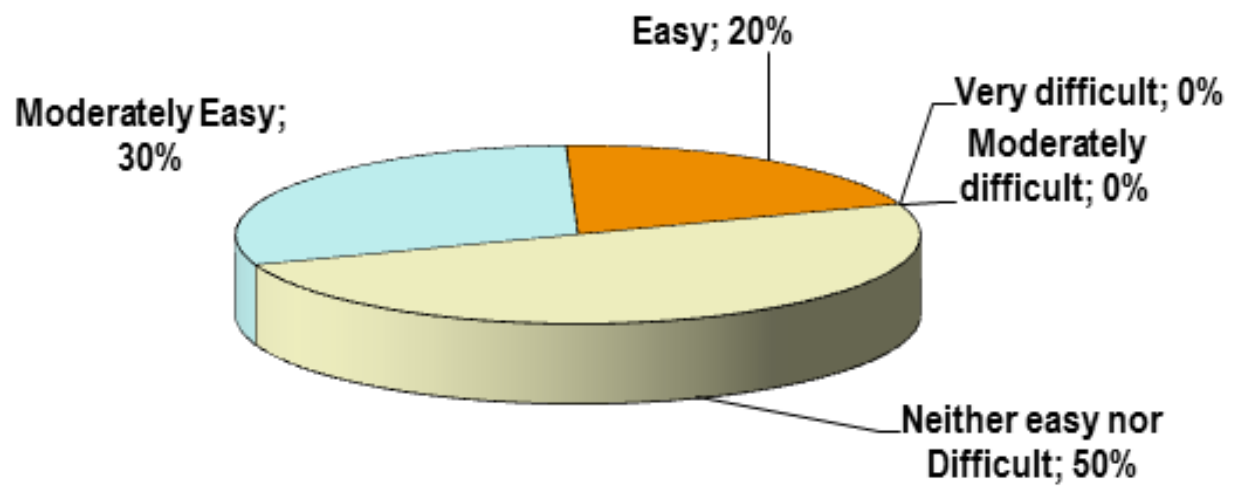

Figure 14: Difficulty level in Task Scenario 4

All of the users mentioned "the searching process/typing key words" as the easiest thing to do or understand. It is therefore safe to assume that the participants of the test find the search panel easy to use and thus this feature contributes to the usability of the NP-DVD.

Eighty percent mentioned "problem looking for the front page" as difficult to do or understand while the rest cited "none" (see Figure 15). It is safe to assume that the 20 percent who mentioned that they do not have difficulty in performing Task 4 are those who rated NP-DVD Task 4 as easy. These respondents easily thought of going directly to page column and look for page 1 while the others open a page of the newspaper and manually navigated until they reach the front page.

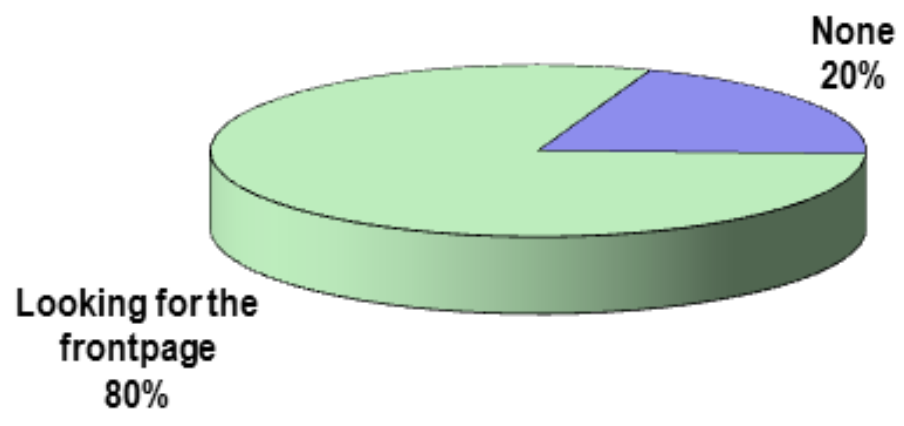

Figure 15: Difficult thing to do or understand

Four participants mentioned they will not change the system to make the task easier while the rest cited searching by page or date range, cropping, and navigation buttons using left and right arrow beside the image of the newspaper (see Figure 16). 


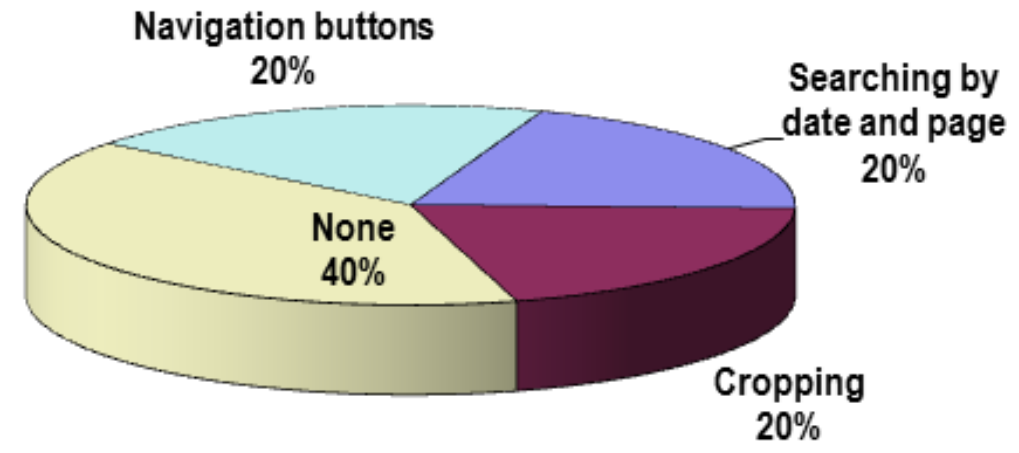

Figure 16. Changes to be made to make Task 4 easier

User 2 completed the 16 activities (i.e., Open, Advance Search, Date, Open front page, Enlarge, Crop, Write, Save, Date, Open front page, Enlarge, Crop, Write, Save, Copy for Disk, and Close) with an average time 0:05:55 minutes, while the slowest were Users 4 and 8 with an average time of 0:06:45 minutes. Other respondents' average time ranges from 0:06:05 to 0:06:30 minutes. It can be assumed that task times of the respondents were still moderate, considering the number of activities needed to accomplish the task almost doubled. The respondents consumed an average of 50 seconds locating the first front page of the newspaper and 36 seconds for the second instance. The time totaled to an average of $1: 26$ or 22.87 percent of the total average time consumed for the whole task. It is also important to note that on the second time the respondent located the front page, the average time was drastically reduced.

The outcome showed that the representative users can make full use of the system on their own. Four among the ten users got a zero error rate in performing the activities, while one respondent got the highest error rate with an error rate of 0.44 . They used several strategies to achieve their goal with minimal error rate. And it is also good to note that the respondents did not encounter errors in loading the DVD, thus it is assumed that at this time, they already know the behavior of the DVD drive in loading and reading the contents. The average memorability rate ranged from "very proficient" to "extremely proficient."

\section{Task Scenario 5: To test if the user can make full use of the system using their style} They were asked to search for an article on a topic they choose. No activities were prepared so that they can be as flexible as possible and perform the action as they desire. Aside from making full use of the system, the test also wanted to know what activities the participants will take and what they will render unimportant, and thus skip such activity.

Sixty percent rated Task 5 as "moderately easy" and 40 percent rated it "easy." This shows that all of them can use the NP-DVD with ease even without a guide (see Figure 17). By reviewing the activities performed by the respondents, we can say that they completed their task using basic search. 


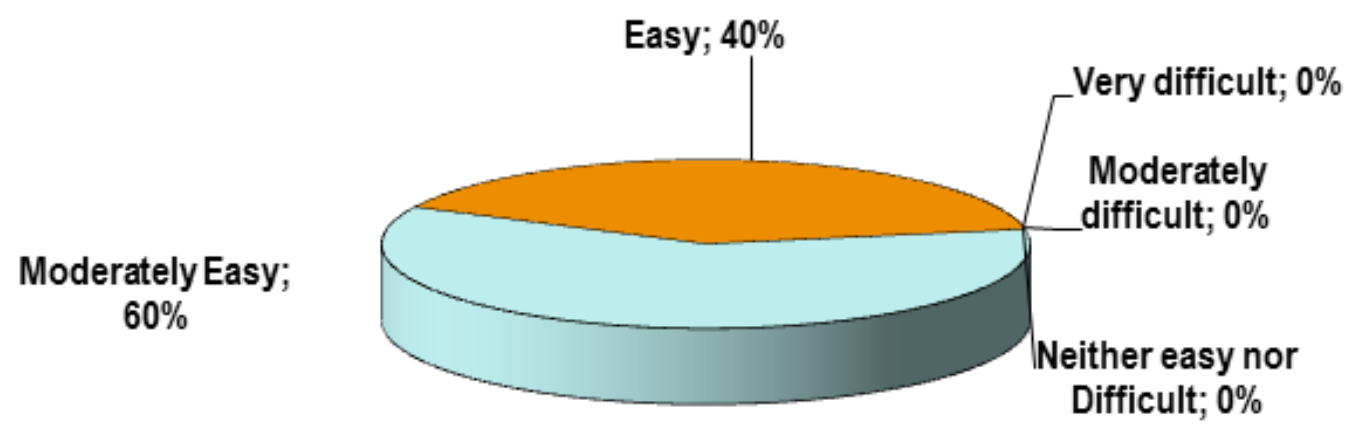

Figure 17: Difficulty level in performing Task 5

All of them mentioned "the searching process/typing key words" as easy. This result shows that the respondents can perform search using their own strategy without difficulty. Fifty percent mentioned "the problem in loading the DVD" as the hardest (see Figure 18).

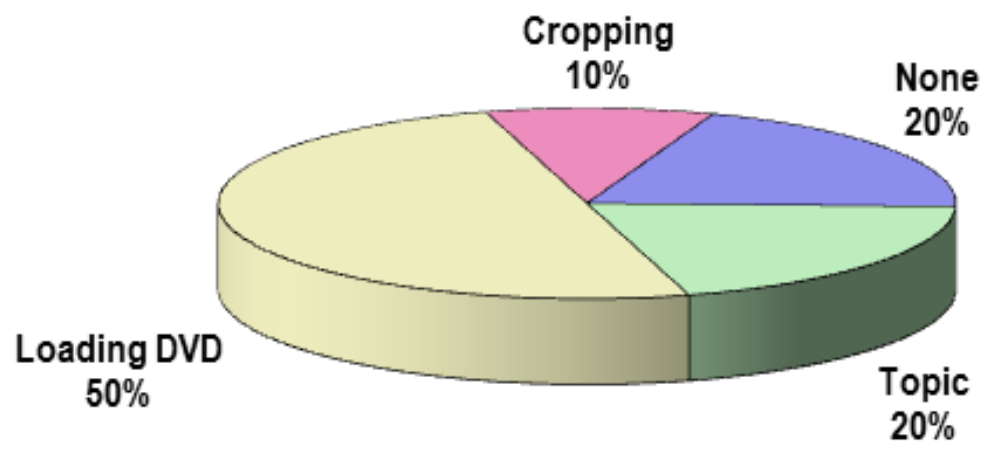

Figure 18. Difficult thing to do or understand without a guide

The fastest among the ten users was User 8 with an average time of 0:02:45 minutes in performing the eight activities (Open, Basic Search, Search, Open Article, Getting and Loading, Enlarge, Crop and Save), while the slowest among the respondents were Users 9 and 10 with an average time of 0:03:10 minutes. The rest had time ranges from 0:02:55 to 0:03:05. It can be inferred that the decrease in the average task time were due to the activities they performed to accomplish Task 5. Therefore we can conclude that using basic search is the fastest strategy to and access a newspaper article.

All of them completed the activities. Majority of them got a completion rate of one. It is noticeable that the respondents employed similar strategies (i.e., basic search) to complete the tasks assigned to them. Seven among the ten users got a zero error rate in performing the activities while one respondent got the highest error rate with an error rate of 0.63 . Results can be attributed to their familiarity for using the NP-DVD. The average memorability rate the respondents got range from 4.75 to 5.00 . The result displayed that the activity with the lowest memorability rate is on the loading of DVD. This is due to the fact the system gave different error messages as the participants use the system (e.g., cannot locate DVD, read error, wrong DVD, and others). 


\section{Post-Test Evaluation}

Upon completion of the five tasks, respondents were given post-test to give their insights on the system. Majority of the respondents rated it "moderately easy" while 20 percent rated it "neither easy nor difficult" and ten percent rated it "easy" (see Figure 19). This result shows that respondents find the system easy to understand. This result can be validated on how the respondents were able to use the system at once since they are all first-time users. Furthermore, the increase in the respondents' productivity is reflected on the decrease in time consumed as they perform the succeeding task without difficulty.

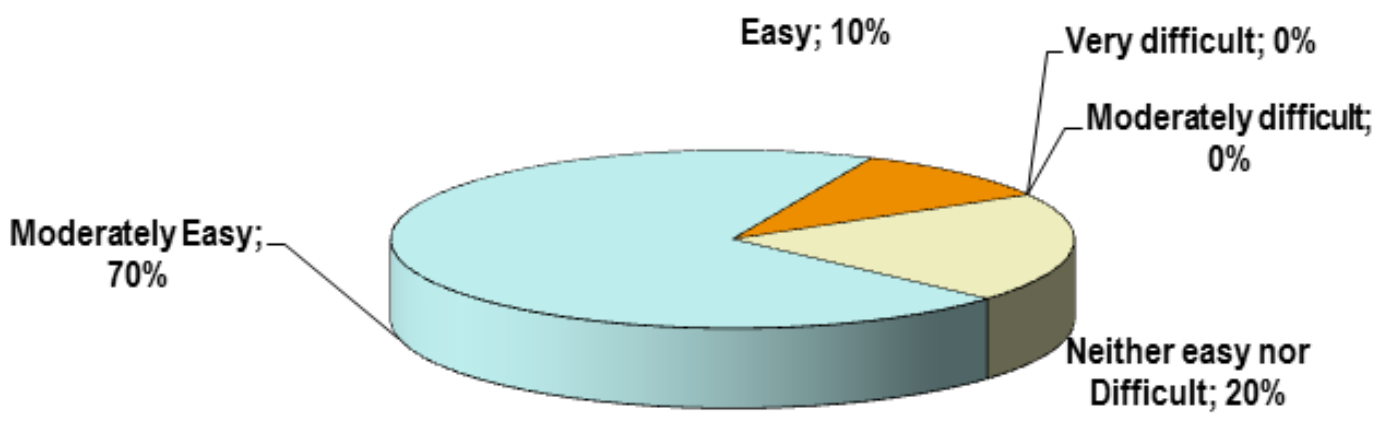

Figure 19. Respondents' rating of ease in using NP-DVD

Majority of the respondents were "satisfied" while 20 percent of them were "very satisfied" (see Figure 20). As one of the attributes of usability this rating will show that if the system is made available in libraries/information centers, it will result to active use of the system in the future.

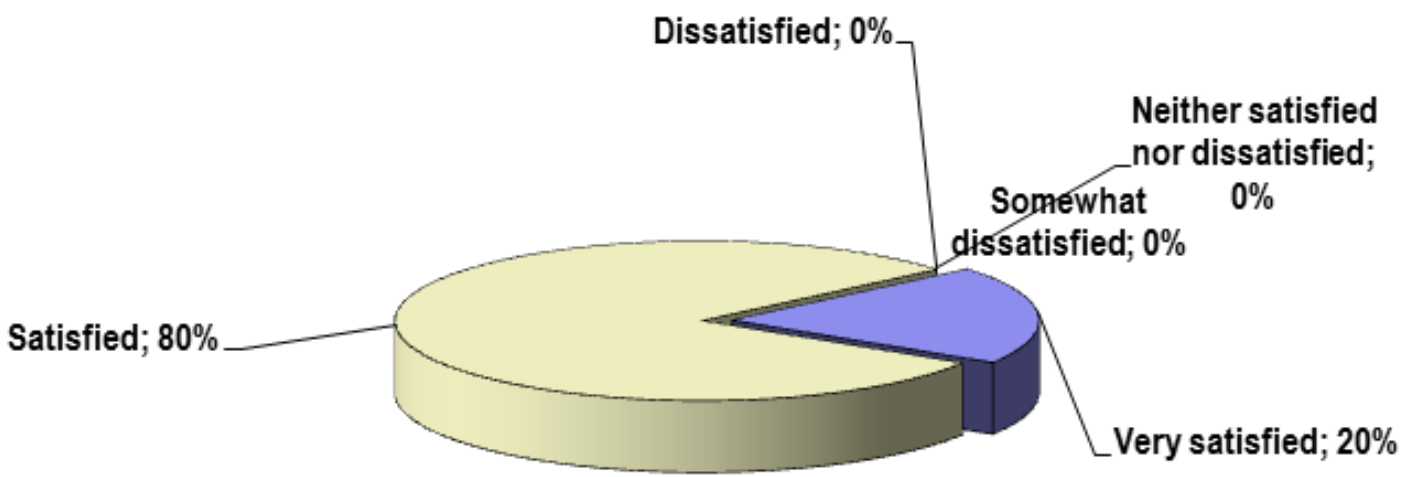

Figure 20: Respondents' overall satisfaction after usability test

The responses show that all the features available in the system is necessary to accomplish the system's objective. All of them will recommend NP-DVD to friends. Two of the respondents mentioned that though the system was a little difficult to use at first after one or two tries you will realize that it is simple and easy to learn. Moreover, $80 \%$ of the respondent felt that the system is very useful in research and locating information that is available in the newspaper. The respondents then suggested adding interactive response, wide side bar, searching by topic and date, and navigation buttons. 


\section{Summary of the Results}

As first-time users in Task 1, some respondents have difficulty in using the system. However, their rating increased as they perform the succeeding task (see Figure 21). This is an important quality of the system since NP-DVD is used only as the users' need arise. Thus, it should be simple and easy to operate so users can use it even without memorizing commands.

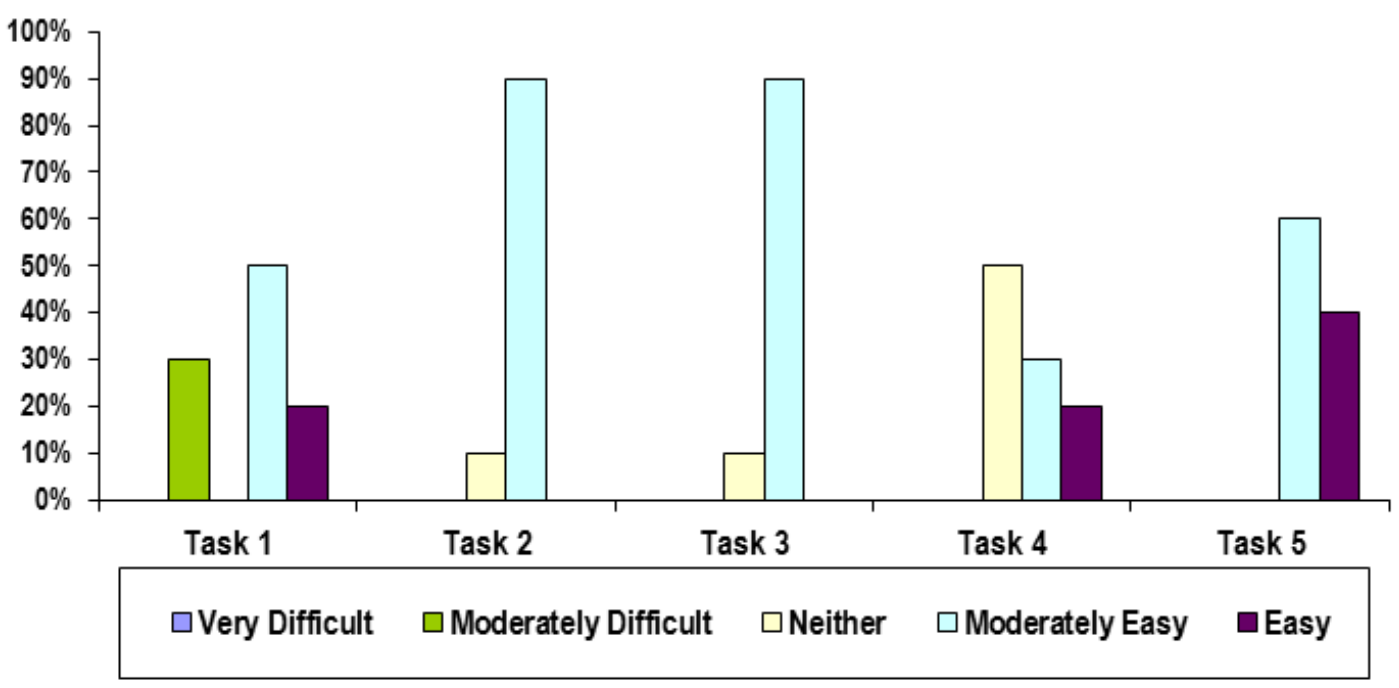

Figure 21. Summary of respondents' rating for ease in using NP-DVD

The summary shows how the respondents were affected by the degree of difficulty of task given to them and their experience in the use of the NP-DVD. The average time for the second task is slightly higher than the first because of addition of two activities. The time decreased in Task 3 is probably because the activities included in Task 2 and 3 are almost the same, thus the procedure taken by the respondents was similar. The increase in the time in Task 4 was due to the increase of the number of activities (from 10 to 16). In Task 5, the decrease of time was most likely due to the decrease in the number of activities and the respondents chose only the activities they already tried in the earlier tasks they had performed (see Figure 22). 


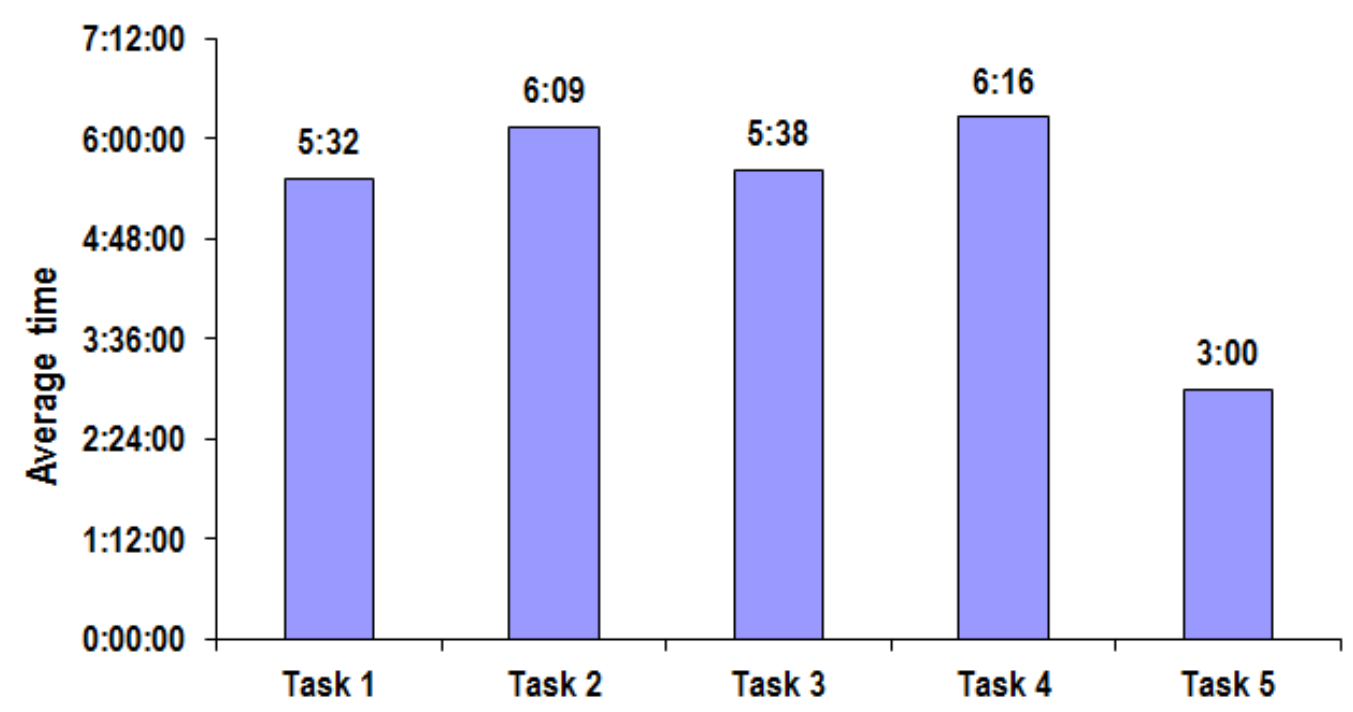

Figure 22: Respondents' average time consumed per task

The test showed that continuously using NP-DVD increases the possibility that respondents will be able to complete all the tasks given to them without asking for assistance (see Figure 23). As experienced in Tasks 1 and 2, one activity was not completed until the library assistant helped the participant (Rate $=3$ ) and two activities were completed only after asking assistance from the library assistant $(R a t e=2)$. On the succeeding tasks the participants completed the activities on their own.

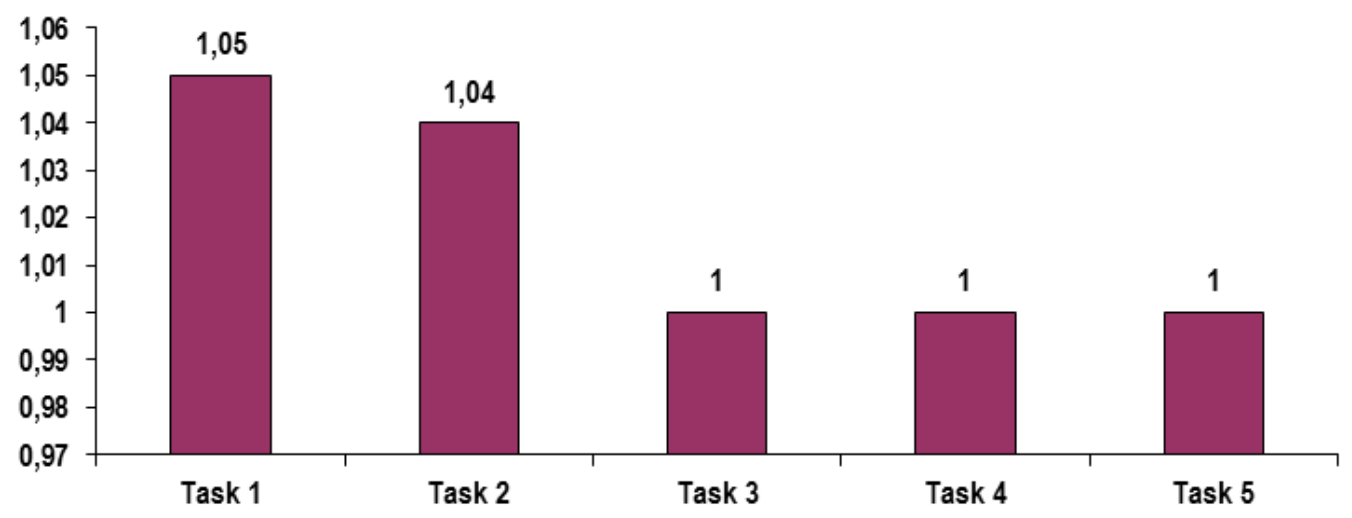

Figure 23: Participants' average completion rate per task

The summary showed that the participants' error rates were affected by the type of activities included in each task. As first time user, the highest error rate was recorded when they performed the first task. The error rate decreased on by 0.06 points when the participants carried out the second task because of new activities included in Task 2. However, the decrease in error rate in Task 3 is noticeable because Tasks 2 and 3 have almost the same activities. Again, the increase in Task 4 was attributed to the inclusion of new and confusing activities. Finally, the decrease again in Task 5 is because of the participants' familiarity with the activities (see Figure 24). The causes of errors are: loading DVDs, printing, typographical errors, cropping process, and saving files. 


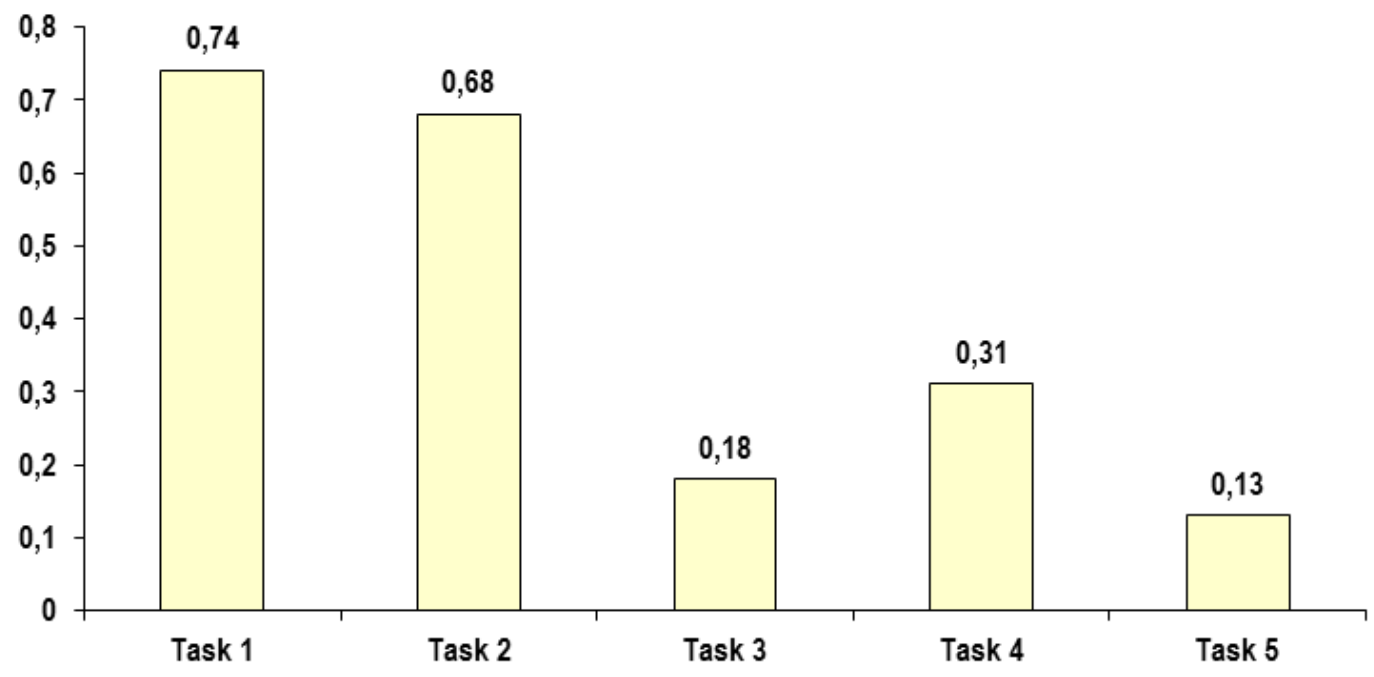

Figure 24: Respondents' average error rate per task

Figure 25 reflects that the participants' memorability rate increases as they perform tasks, whether the tasks were simple or confusing. It is therefore assumed that the memorability rate is greatly affected by the number of times the users use the system. It is believed that the participants started in mid-rate memorability range because of their experience in the use of various computer applications. Thus the increase from 3.29 to 4.95 memorability rating was attributed to the use of the NP-DVD during the usability test.

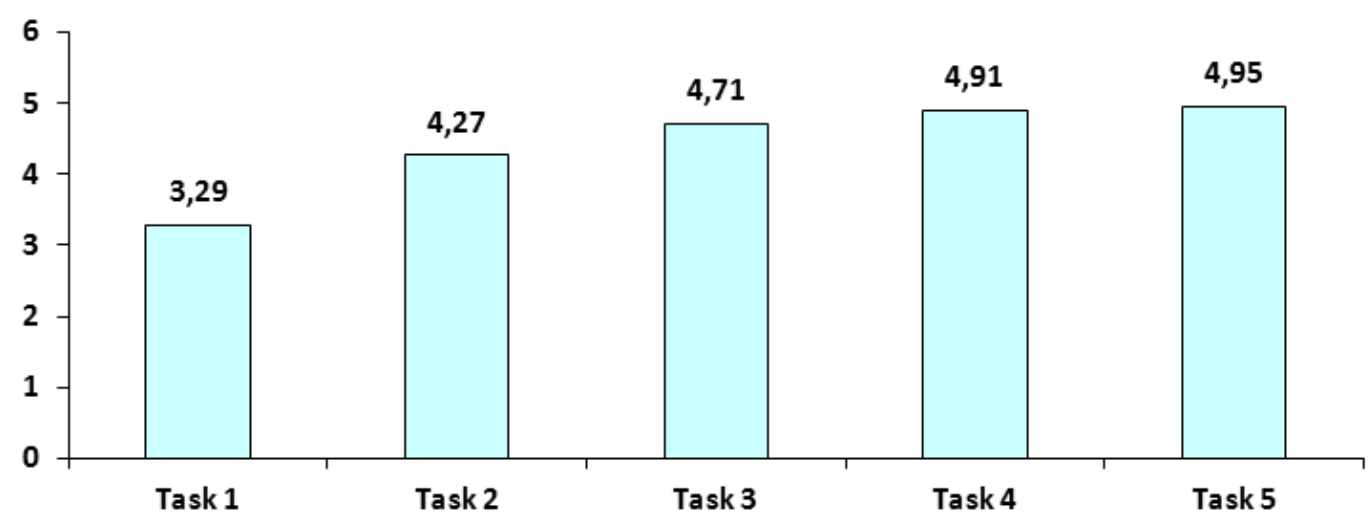

Figure 25: Respondents' average memorability rate per task

\section{Conclusions}

In general, the students find it easy to accomplish the task required of them. The participants learned the system fast enough. The amount of time used by the participants in accomplishing the tasks decreased after the first set. The ability of the students to complete the sets of task reflected a high success rating for the system. Majority of them completed the tasks from Task 1 to 5 . It can be inferred that the respondents got a minimal error rate in using the NP-DVD as evidently seen in the results of error rates in performing Task 1 to 5 .

The following were the recommendations for further development of NP-DVD:

- Rizal Library must conduct an orientation for using NP-DVD. 
- NP-DVD must have interactive responses, wide side bar, an icon for searching by topic and must include navigation buttons.

- The library must study the features that the users like and make possible enhancements. At the same time, the developer must also look at the causes of error and the reasons why the participants experience difficulties and make necessary adjustments.

- Conduct another usability testing in the future using the most recent version to ensure that it is still coping with the new environment.

- Result of the test suggests that the NP-DVD is a product which libraries and other information providers can benefit from. Thus, it should be promoted and marketed.

\section{References}

Landauer T. 1995. "The trouble with computers: usefulness, usability, and productivity." Cambridge, U.K.: MIT Press.

Lacanieta, C. 2000. "Exploring alternative funding models for sustained preservation activity: A history of the self-sufficiency model," in Proceedings of the International Meeting on Microfilm Preservation and Conservation Practices in Southeast Asia:

Assessing Current Needs and Evaluating Past Projects, February 21-24, 2000, Thailand: Chiang Mai University, p. 191-206.

Lewis, J. 2008. "The factor structure of the system usability scale," in HCD 09 Proceedings of the 1st International Conference on Human Centered Design. Berlin, Heidelberg: $\mathrm{HCl}$ International.

Mariano, B.C. 2005. "An Evaluation of the Computerized Index to Philippine Periodical Articles (CIPPA)." Unpublished MLIS special problem, University of the Philippines, Institute of Library and Information Science.

Nielsen J. 2000. "Why you only need to test with five users." Neilsen Norman Group. Retrieved from http://www.nngroup.com/articles/why-you-only-need-to-test-with-5users/.

Neilsen J. 2012. "Usability 101: introduction to usability." Neilsen Norman Group. Retrieved from http://www.nngroup.com/articles/usability-101-introduction-to-usability/

Ronan, L. 2006. "International Coalition on Newspapers." The center for Research Libraries: Focus 26 (1) p. 3-4.

United States Newspaper Project 2005. "National Endowment for the Humanities (NEH): United States Newspaper Program." Retrieved from http://www.Neh.gov/projects/usnp.html.

\section{Biographical note}

Engracia Señga SANTOS finished her Bachelor of Science, major in Computer Engineering, at the Polytechnic University of the Philippines. She received her Masters of Library and Information Science at the University of the Philippines. For fifteen years, she was the head of the Information Communication Technology Unit of the Institute of Philippine Culture, a 
social science research organization of the Ateneo de Manila University. During this time, she co-managed the special library collection of the Institute. In 2007, she transferred to Rizal Library to help build the digital collection. She is now the Head Librarian at the American Historical Collection of Rizal Library. Her projects focused on digital curation and preservation of historical and rare materials in the collection. She is also member of the website and communications committee of the library. 